\title{
Force measurement in the presence of Brownian noise: Equilibrium-distribution method versus drift method
}

\author{
Thomas Brettschneider, ${ }^{1}$ Giovanni Volpe, ${ }^{1,2, *}$ Laurent Helden, ${ }^{1}$ Jan Wehr ${ }^{3}$ and Clemens Bechinger ${ }^{1,2}$ \\ ${ }^{1}$ Physikalisches Institut, Universität Stuttgart, Pfaffenwaldring 57, D-70550 Stuttgart, Germany \\ ${ }^{2}$ Max-Planck-Institut für Intelligente Systeme, Heisenbergstraße 3, D-70569 Stuttgart, Germany \\ ${ }^{3}$ Department of Mathematics, University of Arizona, Tucson, Arizona 85721-0089, USA
}

(Received 13 September 2010; published 15 April 2011)

\begin{abstract}
The study of microsystems and the development of nanotechnologies require alternative techniques to measure piconewton and femtonewton forces at microscopic and nanoscopic scales. Among the challenges is the need to deal with the ineluctable thermal noise, which, in the typical experimental situation of a spatial diffusion gradient, causes a spurious drift. This leads to a correction term when forces are estimated from drift measurements [G. Volpe, L. Helden, T. Brettschneider, J. Wehr, and C. Bechinger, Phys. Rev. Lett. 104, 170602 (2010)]. Here we provide a systematic study of such an effect by comparing the forces acting on various Brownian particles derived from equilibrium-distribution and drift measurements. We discuss the physical origin of the correction term, its dependence on wall distance and particle radius, and its relation to the convention used to solve the respective stochastic integrals. Such a correction term becomes more significant for smaller particles and is predicted to be on the order of several piconewtons for particles the size of a biomolecule.
\end{abstract}

DOI: 10.1103/PhysRevE.83.041113

PACS number(s): 05.40.-a, 07.10.Pz

\section{INTRODUCTION}

The precise measurement of small forces plays a central role in science and technology. Apart from the instrumental challenge of measuring forces in the piconewton and femtonewton ranges, the underlying concept of how forces are determined between macroscopic objects cannot simply be scaled down to the microscale and nanoscale levels. This is easily understood by considering, e.g., a micron-sized object suspended in a liquid environment. Due to its Brownian motion resulting from collisions with the solvent's molecules inertial effects become largely negligible and the trajectory will look rather different compared to macroscopic objects $[1,2]$. Due to the irregularities of this type of motion, various possible mathematical descriptions for the trajectory of a microscopic object exist, e.g., the stochastic differential equations suggested by Itô and Stratonovich. Under many conditions all these descriptions lead to the identical physical interpretation. This is no longer true when the diffusion coefficient of the suspended particle becomes position dependent, as this typically occurs close to other particles or to a wall. In such cases, different mathematical interpretations are not identical and at most one correctly describes the physical reality. Although the need for such corrections-usually referred to as "spurious drift"has been realized several decades ago, e.g., for numerical simulations [3-6], they are seldom applied when analyzing experimental data $[7,8]$. While such corrections are often small, we have recently demonstrated that, in the presence of a spatial dependence of the diffusion coefficient (a situation often encountered, e.g., in biophysical system) the negligence of the correct spurious drift term can lead not only to the wrong amplitude but even to the wrong sign of the forces acting on a micron-sized object suspended in a liquid environment [9].

In this paper we provide a systematic study of how the presence of a position-dependent diffusion coefficient alters

\footnotetext{
*g.volpe@ physik.uni-stuttgart.de
}

the interpretation of the forces acting on a colloidal particle. By extending the results we presented in Ref. [9] we show that only when the correct spurious drift term is subtracted are the measured forces in agreement with theoretical predictions. In particular, we show that the magnitude of the spurious forces increases when considering smaller particles, as often employed in biophysical experiments: The spurious forces acting on a biomolecule with a diameter of about $10 \mathrm{~nm}$ can be on the order of several piconewtons. This value is comparable to the elastic forces that are commonly found in experiments probing the biomechanics of single molecules $[10,11]$.

In Sec. II we discuss the physical origin of the mathematical ambiguity in the description of Brownian motion in a diffusion gradient and how this ambiguity may result in different formulas for the estimation of a force from experimental data. We also show that there are essentially two ways of measuring forces at the nanoscale level. The first is based on the equilibrium distribution of a particle (or particles) subjected to an a priori unknown force. The second takes advantage of the fact that an applied force results in a drift of the particle. In Sec. III we provide some detailed information on the total internal reflection microscopy (TIRM) technique and the materials we employed. In Sec. IV we report the results of the force measurements on particles of various sizes and materials suspended in water close to a wall under the action of electrostatic forces and effective gravity, quantifying in each case the value of the spurious forces. Finally, in the Appendix we clarify how to describe correctly such phenomena using both stochastic differential equations and Fokker-Planck equations.

\section{THEORY: FORCE MEASUREMENTS IN AN OVERDAMPED SYSTEM}

The forces acting on a microscopic object immersed in a fluid medium can be assessed either by studying the underlying 


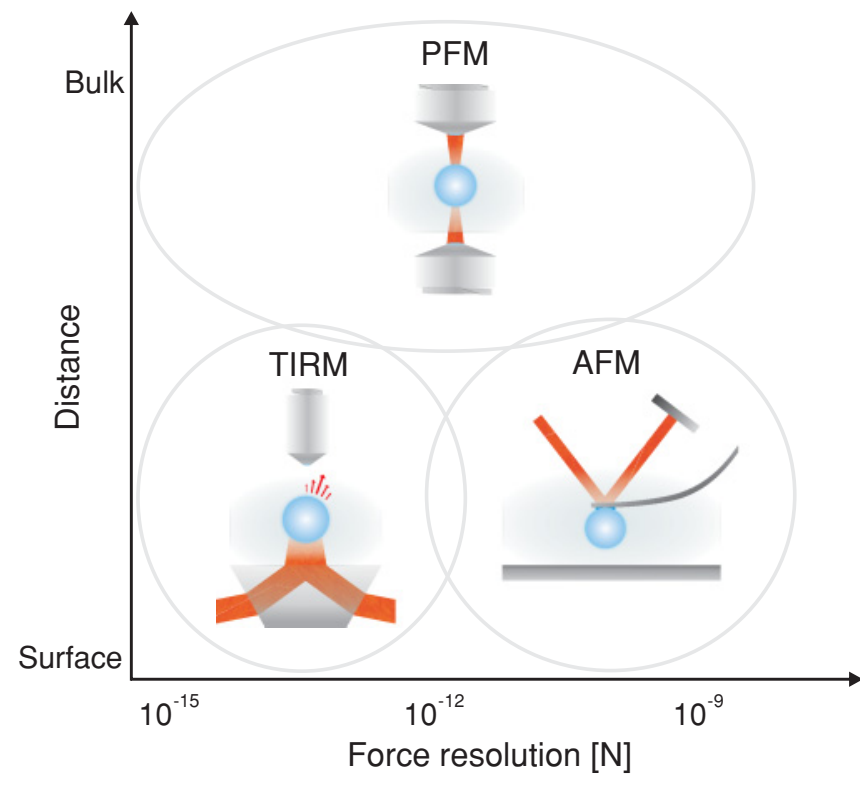

FIG. 1. (Color online) Main techniques of measuring forces at the microscopic and nanoscopic scales, classified according to their force resolution and the working conditions-surface and bulkfor which they are best suited: atomic force microscopy (AFM) [12], photonic force microscopy (PFM) [13-15], and total internal reflection microscopy (TIRM) [16-18].

potential or by studying their effect on the object's trajectory (for an overview of force-measurement techniques and their force resolution, see Fig. 1). The first approach-which we shall refer to as the "equilibrium distribution method"requires sampling of the equilibrium distribution. Accordingly, it can be applied only under conditions where the investigated system is at or close to thermodynamic equilibrium with a heat bath. The second method-which we shall refer to as the "drift method"- - does not require the object to be at or even close to thermal equilibrium. Therefore, it can be applied also to systems that are intrinsically out of equilibrium, e.g., molecular machines, transport through pores, and DNA stretching $[10,11]$. The latter method, however, requires the detection of the object trajectory with high sampling rates, which can be technologically more challenging, in particular when combined with a high spatial resolution.

\section{A. Equilibrium-distribution method}

A microscopic object in contact with a thermal heat bath at constant temperature does not come to rest, but keeps on jiggling around due to the presence of thermal agitation. When the particle is subjected to an external potential well $U(z)$, this leads to a Boltzmann position distribution $p(z)=$ $\exp \left\{-\left[U(z) / k_{B} T\right]\right\}$, where $k_{B}$ is the Boltzmann constant and $T$ is the temperature of the heat bath.

Accordingly, it is possible to sample the steady-state position probability distribution $p(z)$ by measuring a large number of uncorrelated object positions [Fig. 2(a)]. The equilibrium potential can be derived from $U(z)=-k_{B} T \ln [p(z)]$ [Fig. 2(b)] and the force [Fig. 2(c)] from

$$
F(z)=-\frac{d U(z)}{d z}=\frac{k_{B} T}{p(z)} \frac{d p(z)}{d z} .
$$

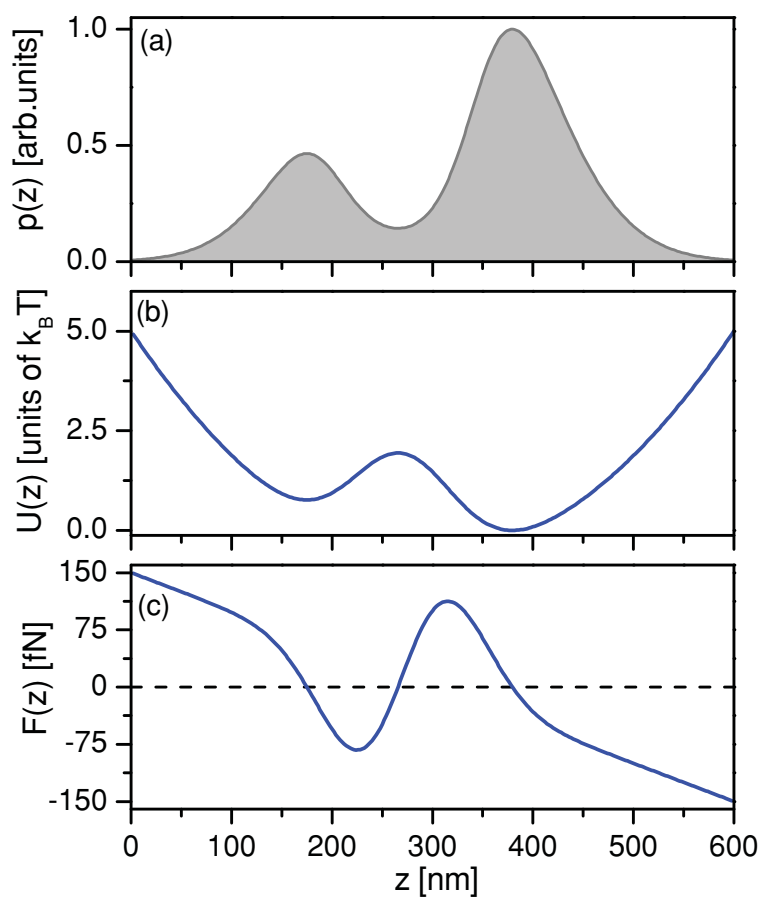

FIG. 2. (Color online) Schematic procedure for measuring a force $F(z)$ by the equilibrium-distribution method. From (a) the measured equilibrium probability distribution $p(z)$ of a particle one obtains (b) the potential-energy distribution $U(z)=-\ln p(z)$, which then gives (c) the force $F(z)=-\frac{\partial}{\partial z} U(z)$ [Eq. (1)].

Due to the exponential dependence of the probability distribution on the potential depth, in typical experiments, only potential minima within typically less than approximately $5 k_{B} T$ are explored by the particle. While Eq. (1) can be applied only to equilibrium conditions, it should be mentioned that recently it has been demonstrated that for the specific case of nonequilibrium steady states a similar relation, i.e., a generalized Boltzmann distribution, can be derived for the stationary particle equilibrium distribution and the conservative part of the potential $[19,20]$.

\section{B. Drift method}

Since for a microscopic body suspended in a liquid medium viscous forces prevail by several orders of magnitude over inertial effects, a constant force $F$ applied to a microscopic particle results in a constant drift velocity $v=F / \gamma$, where $\gamma$ is the object's friction coefficient. Since $v=\Delta z / \Delta t$ can be retrieved from the measured particle displacement $\Delta z$ within time $\Delta t$, the force can be measured accordingly as

$$
F=\gamma \frac{\Delta z}{\Delta t} .
$$

For large forces this obviously leads to a univocal result. However, when the drift force amplitude is comparable to the effect of the thermal noise, the measured particle displacement $\Delta z$ and thus the drift force vary between identical experiments, 


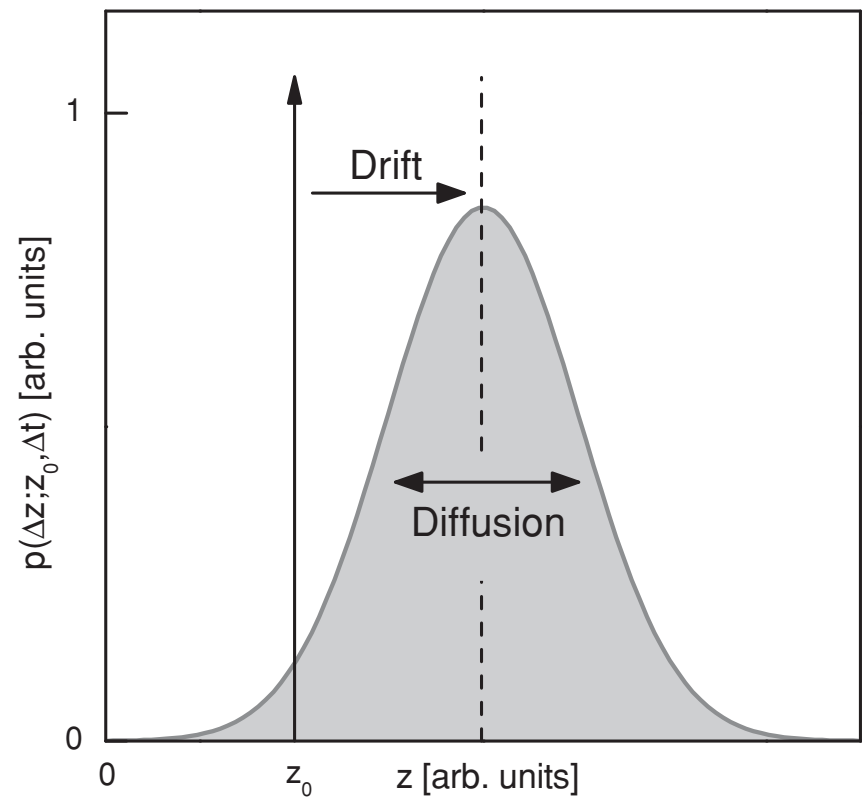

FIG. 3. Schematic view of the propagator $p\left(\Delta z ; z_{0}, \Delta t\right)$, which gives the distribution of the particle position increments $\Delta z$ from its initial position $z_{0}$ after a time step $\Delta t$. For sufficiently small $\Delta t$ the distribution is gaussian with standard deviation $\sqrt{2 D z_{0} \Delta t}$ [Eq. (4)]. The force can be estimated from the measured drift according to Eq. (5).

leading to a statistical distribution of the measured values (Fig. 3):

$$
F(z)=\gamma\left\langle\frac{\Delta z_{j}(z)}{\Delta t}\right\rangle,
$$

where $\Delta z_{j}(z)$ denotes the $j$ th experimental value of the particle's displacement after time $\Delta t$.

Although Eq. (3) is key to measuring forces under nonequilibrium conditions, it is valid only in situations where the diffusion coefficient $D=k T / \gamma$ of the object to which the force is applied is constant. When $D$ becomes position dependent, i.e.,

$$
D(z)=\left\langle\frac{\left[\Delta z_{j}(z)-\left\langle\Delta z_{j}(z)\right\rangle\right]^{2}}{2 \Delta t}\right\rangle,
$$

Eq. (3) must be corrected by an additional term $[9,21]$

$$
F(z)=\gamma(z)\left\langle\frac{\Delta z_{j}(z)}{\Delta t}\right\rangle-\alpha \gamma(z) \frac{d D(z)}{d z},
$$

which we shall refer to as "spurious force" and which may depend on the specific choice of $\alpha \in[0,1]$.

In the case of systems that are coupled to a heat bath, thermodynamic consistency requires that $\alpha=1$ [22]. In particular, this is true for a Brownian particle, which is the system we have experimentally investigated. More generally, other values of $\alpha$ might be possible when describing other stochastic processes [23].

\section{Physical origin of the spurious force}

A qualitative physical understanding of the correction term in Eq. (5) can be gained by considering the effect of a diffusion

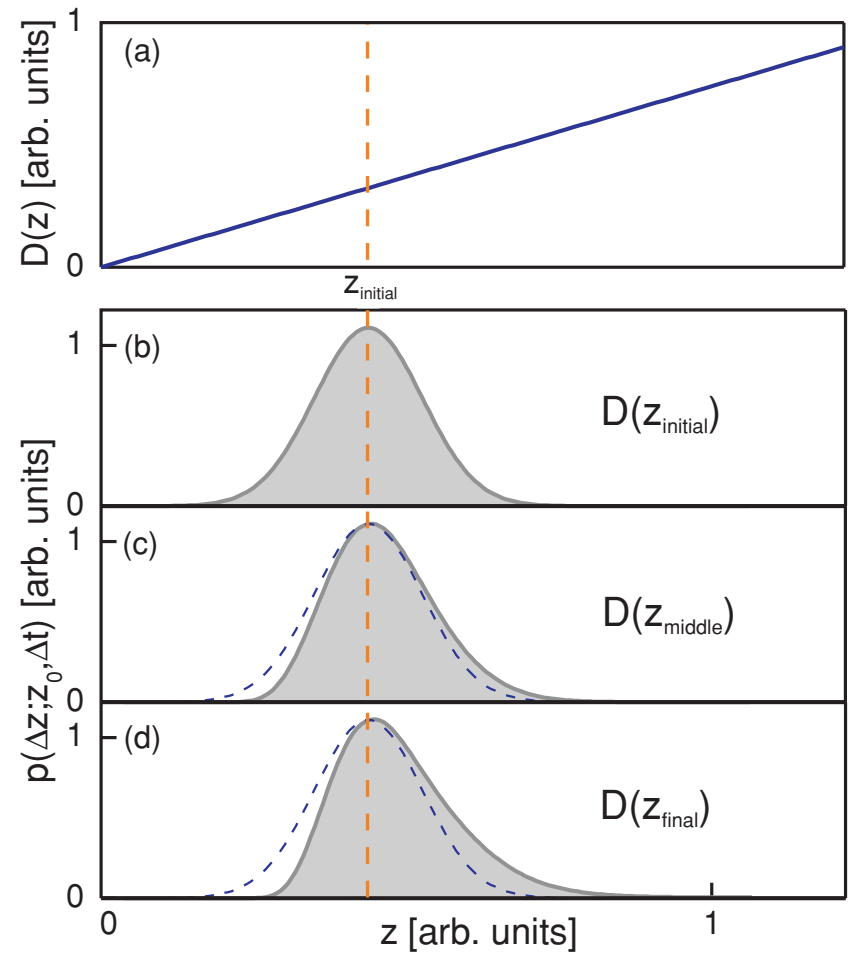

FIG. 4. (Color online) In the presence of (a) a diffusion gradient, the propagator $p\left(\Delta z ; z_{0}, \Delta t\right)$ depends on where $D$ is evaluated, i.e., at (b) $z_{\text {initial }}$, (c) $z_{\text {middle }}$, or (d) $z_{\text {final }}$. In the latter two cases this results in a shift of the position distribution mean-the spurious drift.

gradient [Fig. 4(a)] on a Brownian particle initially localized at position $z_{\text {initial }}$ (verticaldashed line in Fig. 4) at time $t_{0}$.

In the simplest picture, the particle diffusion results in a dichotomic movement either to the left or to the right with the same probability; therefore, after time $\Delta t$ the particle is displaced by $z_{\text {initial }} \pm \sqrt{2 D} \Delta t$. In a more realistic picture, the particle's final position has a continuous probability distribution. In both cases, by assuming $D$ is constant, the final particle position distribution $p\left(\Delta z ; z_{0}, \Delta t\right)$ is symmetric, like the histogram of Fig. 4(b).

In the presence of a diffusion gradient [Fig. 4(a)] the value of $D$ is obviously different at the initial and final positions; therefore the evaluation of the displacement is not univocal. If we assume that $D=D\left(z_{\text {intial }}\right)$-which we shall refer to as the "Itô convention" $-p\left(\Delta z ; z_{0}, \Delta t\right)$ is symmetric, as in the constant diffusion coefficient case [Fig. 4(b)]. However, it could be argued that $D$ should be averaged over the particle displacement; if we assume thus $D=D\left(z_{\text {middle }}\right)-$ the "Stratonovich convention"- $p\left(\Delta z ; z_{0}, \Delta t\right)$ becomes asymmetric [Fig. 4(c)] because the particle displaces further when moving toward increasing diffusion. Finally, if we assume $D=D\left(z_{\text {final }}\right)$ — the "isothermal, anti Itô or backward Itô convention" $-p\left(\Delta z ; z_{0}, \Delta t\right)$ becomes even more asymmetric [Fig. 4(d)]. The spurious drift and the related spurious force account for such asymmetry.

In more general terms, we might assume $D=(1-$ $\alpha) D\left(z_{\text {initial }}\right)+\alpha D\left(z_{\text {final }}\right)$ and $\alpha \in[0,1]$, with $\alpha=0$ corresponding to the Itô convention, $\alpha=0.5$ to the Stratonovich convention, and $\alpha=1$ to the isothermal convention. The latter 
one in particular turns out to be the only thermodynamically consistent one for a system coupled to a heat bath [22].

\section{METHODS AND MATERIALS}

\section{A. Total internal reflection microscopy}

The trajectories of colloidal particles close to a wall are measured with TIRM [16-18] and a scheme of a typical setup is presented in Fig. 5(a). A $p$-polarized laser beam $(\lambda=658 \mathrm{~nm})$ is totally internally reflected at a glass-liquid interface generating an evanescent field decaying into the liquid. A spherical colloidal particle in the vicinity of the interface scatters the evanescent light. The scattering intensity shows a marked dependence on the particle position $I(z)=$ $I_{0} \exp (-\beta z)$, where $\beta$ is the inverse evanescent decay length and $I_{0}=I$ (with $z=0$ ) [Fig. 5(b)]. The scattering intensity time series $I(t)$ is collected by an objective and recorded by a photomultiplier [Fig. 5(c)] at a sampling rate of $1 \mathrm{kHz}$ for about $200 \mathrm{~min}$. Inverting the position-intensity relation, we finally obtain the particles' trajectory $z(t)$ with a spatial resolution of a few nanometers [Fig. 5(d)]. Since our system is in thermal equilibrium, the measured particle trajectory allows us to calculate the forces via both the equilibrium distribution and the drift method, as described above.
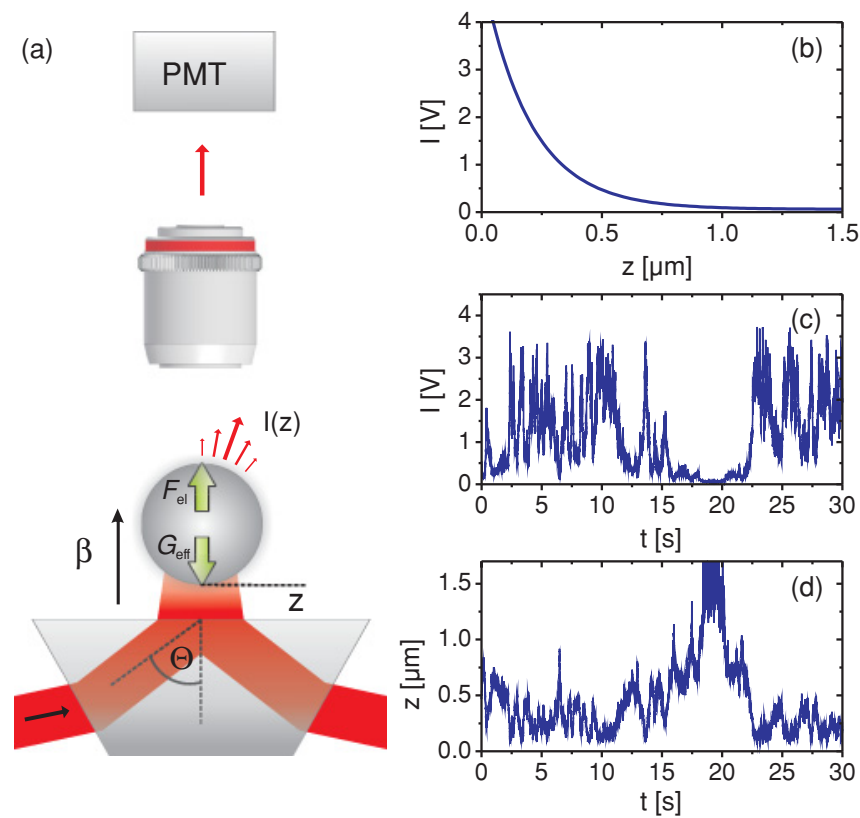

FIG. 5. (Color online) Total internal reflection microscopy. (a) Sketch of a typical TIRM geometry with a single colloidal particle in front of a planar transparent wall. The particle is illuminated by the evanescent field created by the total internal reflection of a laser beam at the glass-fluid interface while undergoing Brownian motion. The scattered intensity $I$ is measured using a photomultiplier (PMT). The forces acting on the particle are due to gravity $G_{\text {eff }}$ and electrostatic interactions $F_{\text {el }}$ and are indicated by opposing arrows. When (b) the relationship between the scattered intensity and distance $I(z)$ is known, (c) the intensity time series $I(t)$ can be converted into (d) the trajectory $z(t)$.

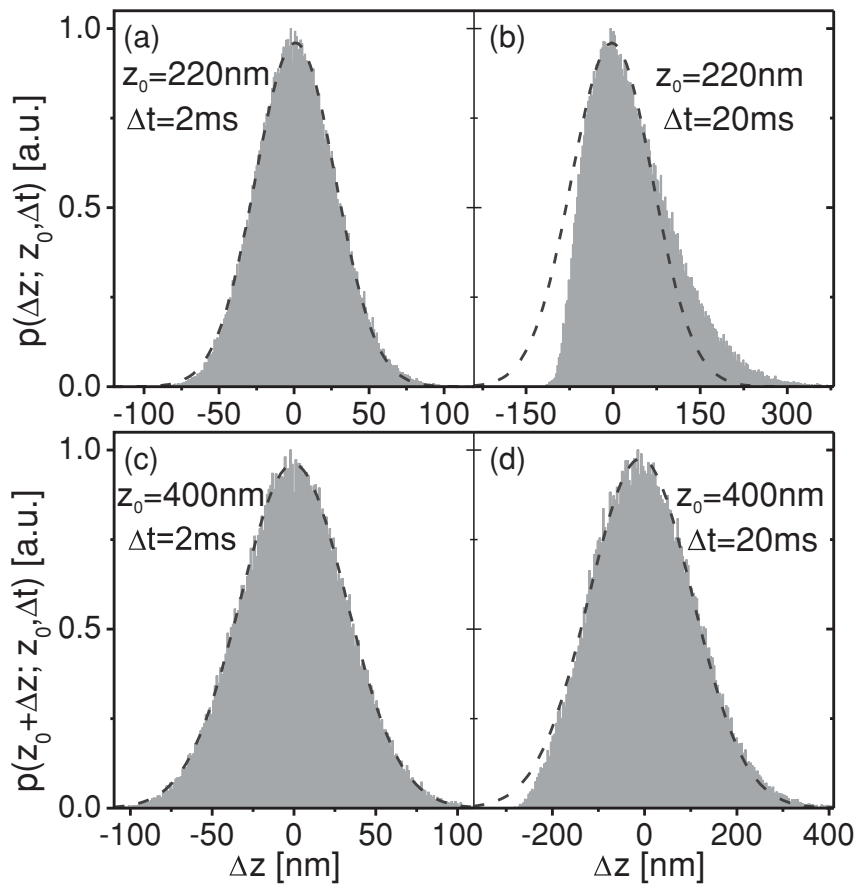

FIG. 6. Influence of $\Delta t$. At the distance $z_{0}=220 \mathrm{~nm}$, (a) $\Delta t=$ $2 \mathrm{~ms}$ leads to a Gaussian distribution of the position increments, but for (b) $\Delta t=20 \mathrm{~ms}$, a clear deviation from a Gaussian distribution appears. At $z=400 \mathrm{~nm}$, where the spatial variation of the drift is much smaller, (c) the distribution is again Gaussian for $\Delta t=2 \mathrm{~ms}$ and (d) remains almost Gaussian also for $\Delta t=20 \mathrm{~ms}$.

Due to thefractal nature of the Brownian motion, the value of $\left\langle\Delta z_{j}(z) / \Delta t\right\rangle$ that has to be calculated for the drift force [see Eq. (5)] strongly depends on the chosen time interval $\Delta t$. On the one hand, $\Delta t$ should be as short as possible; on the other hand, it cannot be made arbitrarily small due to the finite experimental acquisition frequency. A reasonable trade-off is to choose $\Delta t$ so small that the spatial variation of the drift force during such a time step is negligible. In order to meet these conditions in our experiment, we have stepwise reduced $\Delta t$ until the probability distribution of $\Delta z$ became Gaussian, as expected when the above condition holds. Because under our conditions (see Sec. III A) the spatial gradient of the force acting on the particle is not constant, the above condition on $\Delta t$ is expected to vary with the particle-wall distance. This is shown in Fig. 6, where we plotted the probability distribution of $\Delta z$ for $\Delta t=2$ and $20 \mathrm{~ms}$ obtained at two particle-wall distances $z=220$ and $400 \mathrm{~nm}$, which correspond to the distances with the largest and smallest spatial variations of the

TABLE I. Particle and sample parameters. Titanium oxide particles were prepared according to the procedure in Ref. [24]. Melamin particles were acquired from Microparticles GmbH (MFF-1.3). Polystyrene particles were acquired from Invitrogen (IDC 1-2400).

\begin{tabular}{lccccc}
\hline \hline$R(\mathrm{~nm})$ & Material & $\rho_{s}\left(\mathrm{~g} / \mathrm{cm}^{3}\right)$ & $\kappa^{-1}(\mathrm{~nm})$ & $B(\mathrm{pN})$ & $\mathrm{NaCl}(\mu \mathrm{m})$ \\
\hline 400 & titanium -oxide & 2.54 & 25 & 68 & 150 \\
655 & melamin & 1.51 & 18 & 770 & 300 \\
1180 & polystyrene & 1.05 & 18 & 1080 & 300 \\
\hline \hline
\end{tabular}




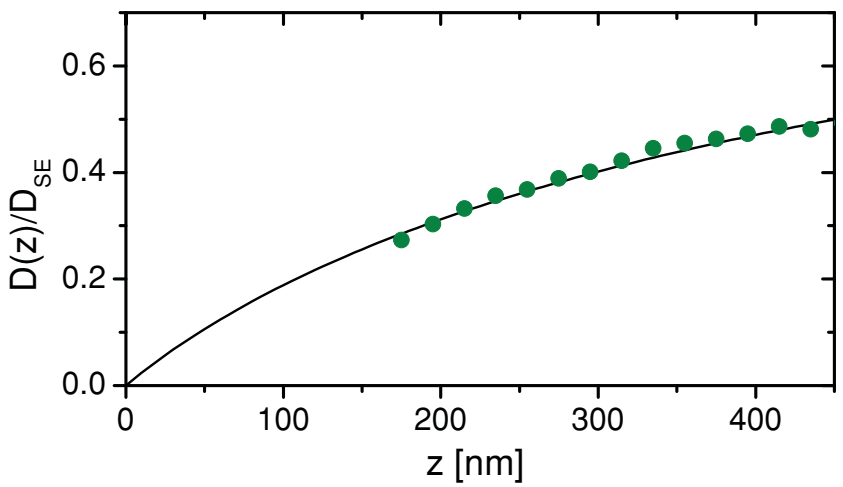

FIG. 7. (Color online) Comparison of measured (solid circles) and calculated (line) normalized vertical diffusion coefficient $D_{\perp} / D_{\mathrm{SE}}$ for an $R=400 \mathrm{~nm}$ particle as a function of the particle-wall separation $z$.

force acting on the particle. As can be seen, for $\Delta t=2 \mathrm{~ms}$, we always obtain a Gaussian distribution, while for $\Delta t=20 \mathrm{~ms}$ deviations from a Gaussian fit (dashed line) are observed. In all measurements presented in the following we have chosen $\Delta t=2 \mathrm{~ms}$.

\section{B. Gravitational and electrostatic forces}

In order to compare the measured forces with theory, it is important to have full knowledge of the interaction mechanisms of a colloidal particle with a wall. For an electrically charged dielectric colloidal sphere suspended in a solvent, the interaction forces have been demonstrated to be described by [16-18]

$$
F(z)=B e^{-\kappa z}-G_{\mathrm{eff}} .
$$

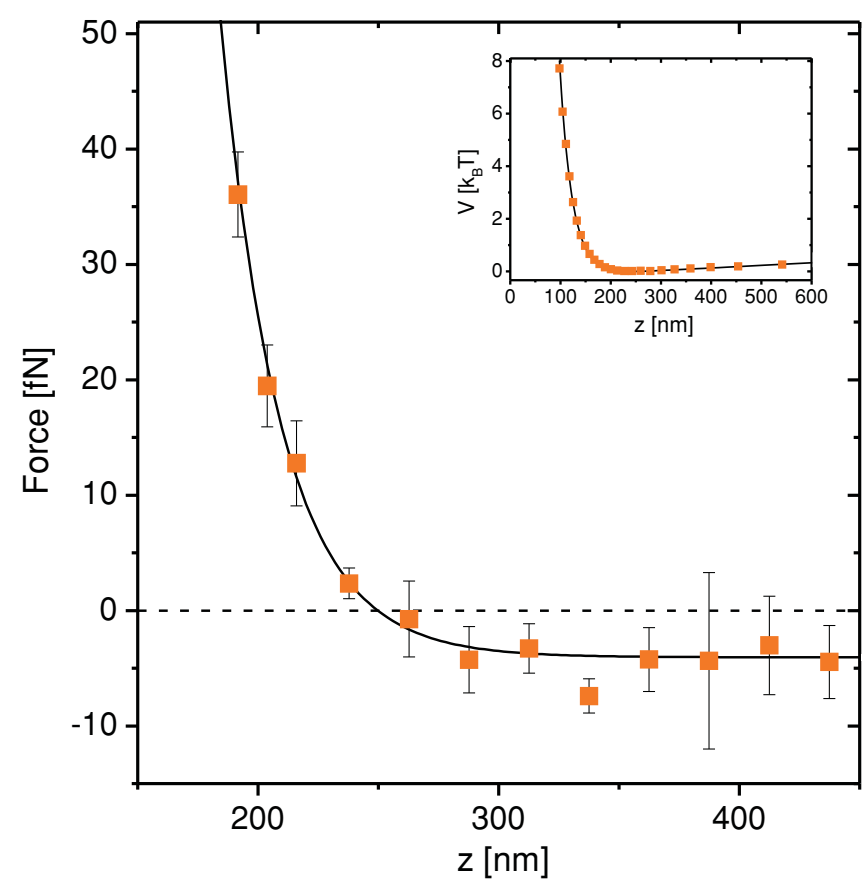

FIG. 8. (Color online) Force derived by the equilibriumdistribution method (squares) for the 400-nm-radius particle (see Table I) and theoretical expectation (line) according to Eq. (6). The inset shows the corresponding potential $U(z)$.

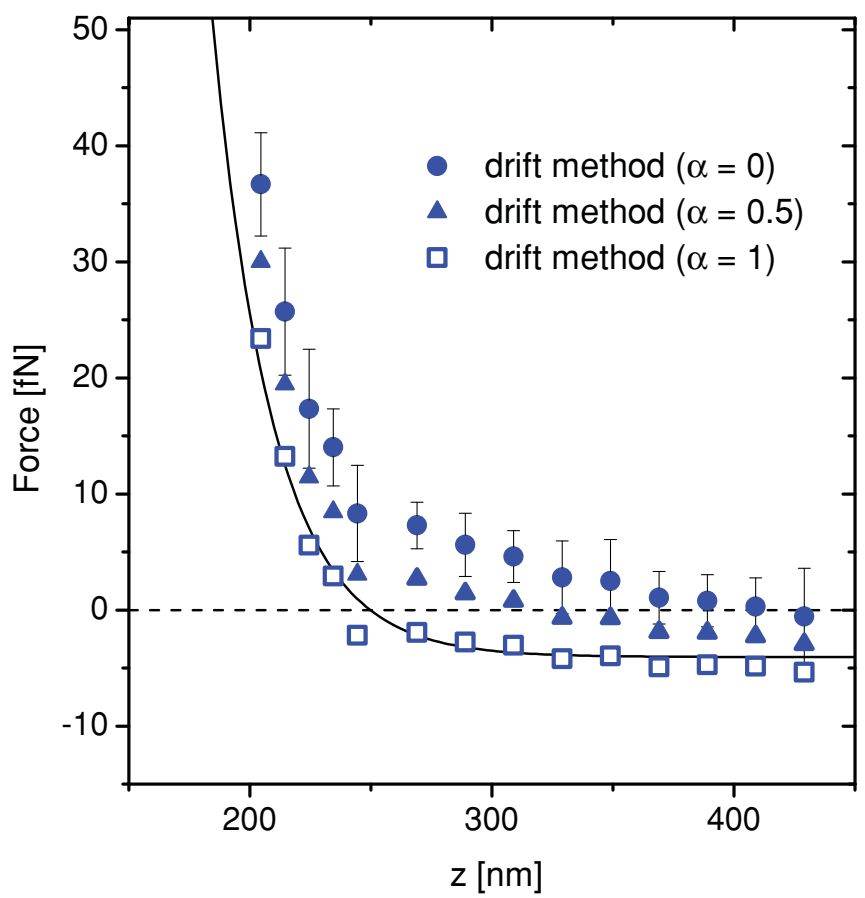

FIG. 9. (Color online) Force derived by the drift method for the very same particle as in Fig. 8. The force measured according to Eq. (5) with $\alpha=1$ (solid circles) coincides with the theory (line), while with $\alpha=0$ (circles) and $\alpha=0.5$ (triangles) there is a clear disagreement.

The first term is due to double layer forces with $\kappa^{-1}$ the Debye length and $B$ a prefactor depending on the surface charge densities of the particle and the wall (see Table I). The second term describes the effective gravitational contributions $G_{\text {eff }}=\frac{4}{3} \pi R^{3}\left(\rho_{p}-\rho_{s}\right) g$ with $\rho_{p}$ and $\rho_{s}$ the particle and solvent density and $g$ the gravitational acceleration constant. Under our conditions the additional contribution of van der Waals forces can be neglected since they become relevant only at much shorter particle-wall distances [25,26].

\section{Diffusion in the bulk and diffusion gradient in front of a wall}

The Stokes-Einstein diffusion coefficient of a spherical colloidal particle immersed in a solvent is $D_{\mathrm{SE}}=k_{B} T / 6 \pi \eta R$, where $\eta$ is the shear viscosity of the liquid. Close to a wall the bulk diffusion coefficient decreases due to hydrodynamic interactions. From the solution of the creeping flow equations for a spherical particle moving near a wall assuming nonslip boundary conditions, the vertical component of the diffusion coefficient is [27]

$$
D_{\perp}(z)=\frac{D_{\mathrm{SE}}}{l(z)},
$$

where

$$
\begin{aligned}
& l(z)=\frac{4}{3} \sinh [a(z)] \sum_{n=1}^{\infty} \frac{n(n+1)}{(2 n-1)(2 n+3)} \\
& \left(\frac{2 \sinh [(2 n+1) a(z)]+(2 n+1) \sinh [2 a(z)]}{4 \sinh ^{2}[(n+0.5) a(z)]-(2 n+1)^{2} \sinh ^{2}[a(z)]}-1\right) \\
& \text { and } \quad a(z)=\cosh ^{-1}\left(1+\frac{z}{R}\right) .
\end{aligned}
$$




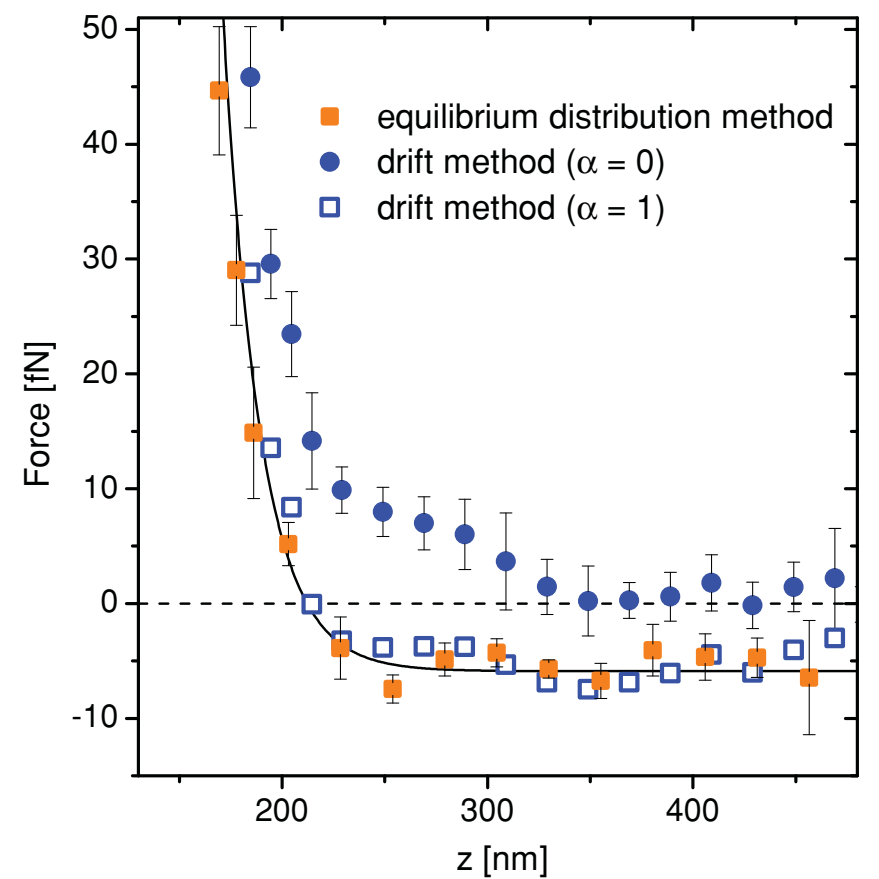

FIG. 10. (Color online) Comparison of forces obtained for the 655-nm-radius particle (see Table I). For the equilibrium-distribution method (solid squares) and drift method with correction term (open squares) the results are in agreement with the theoretical expectation (line) according to Eq. (6). Neglecting the correction term leads to a clear deviation (solid circles). These data are the same as those presented in Ref. [9].

$D_{\perp}(z)$ is zero at the wall and monotonically increases with $z$ approaching the bulk value at a distance of several particle radii away from the wall. When calculating $D_{\perp}(z)$ from the particle trajectories of our TIRM measurements according to Eq. (4), indeed we find good agreement with the theoretical prediction (Fig. 7).

\section{Sample preparation and parameters}

As a sample cell we used a 2-mm-thick cuvette comprising two optical flats separated by a spacer of silicon rubber. The cell was filled with clean deionized water containing a very small number of colloidal particles and $150-300 \mu \mathrm{mM}$ of $\mathrm{NaCl}$ salt to adjust the Debye screening length. In order to vary the spatial gradients of the diffusion coefficient we used particles with different radii $R$ and densities since they sample different ranges of $z / R: R=400 \mathrm{~nm}$ (titanium oxide), $R=655 \mathrm{~nm}$ (melamin), and $R=1180 \mathrm{~nm}$ (polystyrene). For further details see Table I.

\section{RESULTS AND DISCUSSION}

In Fig. 8 we show as solid symbols the force [and in the inset the corresponding potential $U(z)]$ acting on a $R=400-\mathrm{nm}$ titanium oxide particle as obtained by the equilibrium distribution method [Eq. (1)]. Since the forces as determined from this method are unambiguous, they will be considered as the true forces acting on the particle. This is also supported by the fact that the experimental data are in quantitative agreement

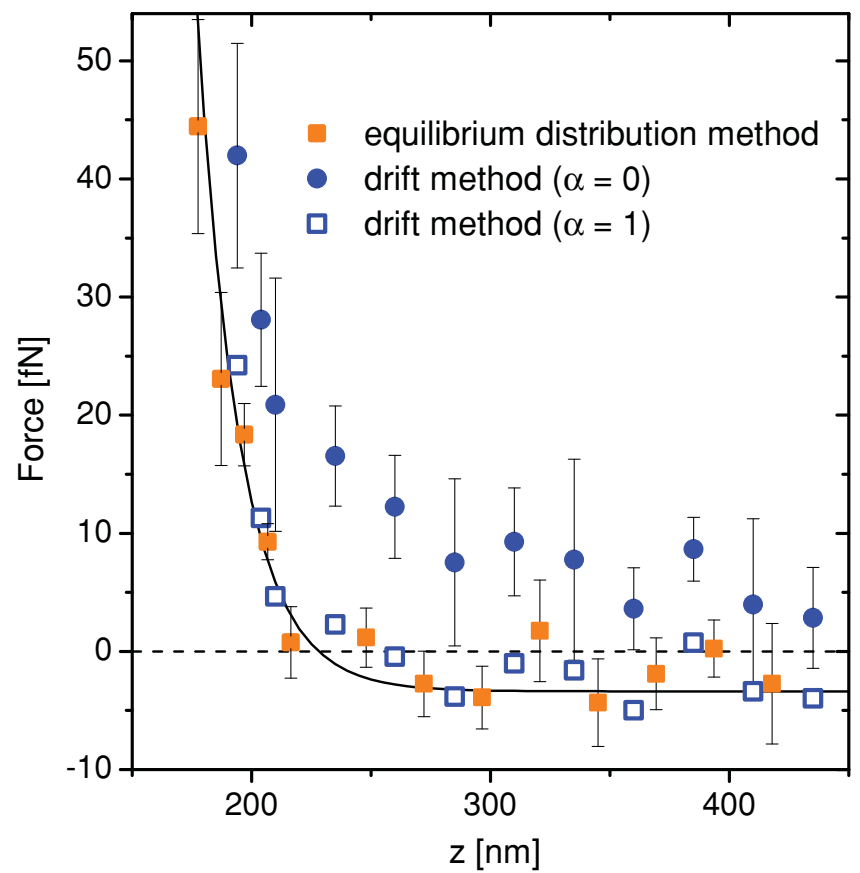

FIG. 11. (Color online) Comparison of forces obtained for the 1180-nm-radius particle (see Table I). For the equilibriumdistribution method (solid squares) and the drift method with the correction term (open squares) the results are in agreement with the theoretical expectation (line) according to Eq. (6). Neglecting the correction term leads to a clear deviation (solid circles).

with Eq. (6) (solid line), where $G_{\text {eff }}$ and $\kappa^{-1}$ are taken from the experimentally known parameters (Table I) while the prefactor $B$ has been treated as a fit parameter. The value $B=68 \mathrm{pN}$ is in agreement with other TIRM measurements under similar conditions [17].

The symbols in Fig. 9 correspond to the force-distance relation as obtained from the drift method using Eq. (5) for $\alpha=0$ (solid circles), $\alpha=0.5$ (triangles), and $\alpha=1$ (open squares). Since the gradient of $D$ vanishes far away from the surface, the force dependence on $\alpha$ is most pronounced close to the wall but weakens at larger $z$ where the curves will eventually merge (only beyond the maximum distance sampled by the particle). The forces determined with $\alpha=1$, i.e., the isothermal convention, show good agreement with Eq. (6) (solid line, the same as in Fig. 8). We want to emphasize that all other choices of $\alpha$, in particular the negligence of the noise-induced correction $(\alpha=0)$, lead to significant differences. Not only the magnitude, but also the sign of the forces obtained with $\alpha=0$ and 0.5 disagrees with the true forces as obtained in Fig. 8.

Similar measurements were also performed with other particles that are capable of sampling even smaller particlewall distances normalized by the particle radius, i.e., $z / R$ (cf. Figs. 12 and 13), where the gradient of the diffusion coefficient becomes larger. Results are shown in Fig. 10 for the $R=655$ $\mathrm{nm}$ particle and in Fig. 11 for the $R=1080-\mathrm{nm}$ particle. In both cases, the equilibrium-distribution measurement (solid squares) agrees with Eq. (6) with $B$ as the only fit parameter and with $\alpha=1$ (open squares). As before, all other choices of 


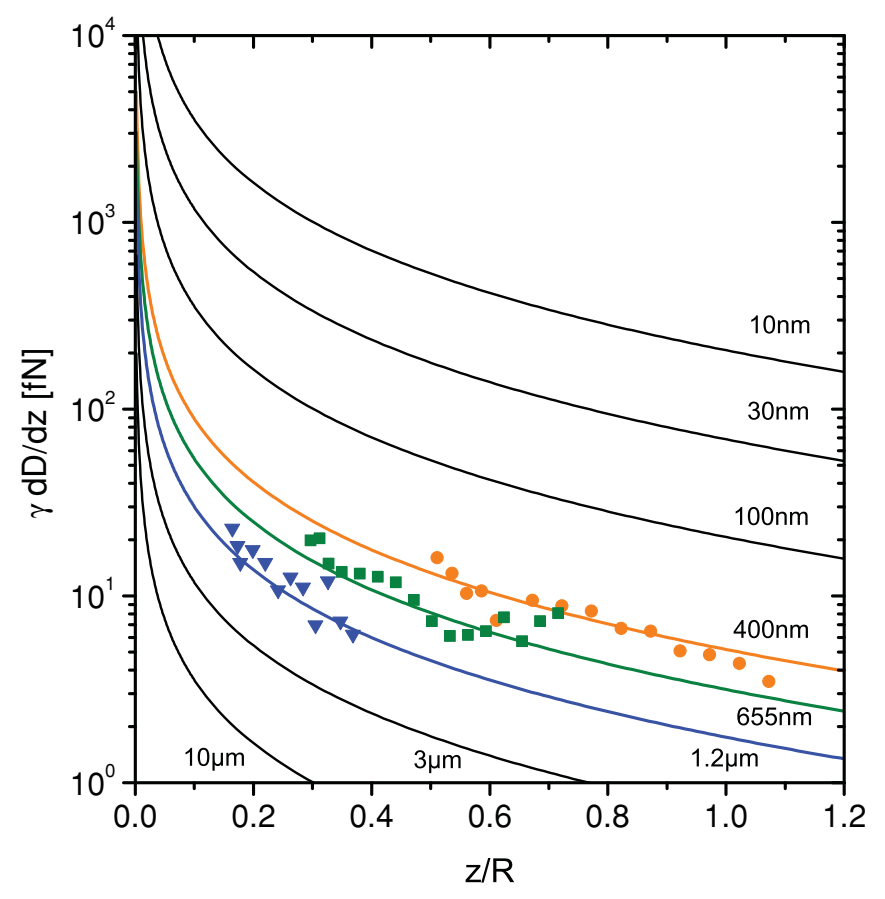

FIG. 12. (Color online) Distance dependence of the theoretically calculated spurious force $\alpha \gamma \frac{d}{d z} D$ with $\alpha=1$ for various particle radii $R$ (solid lines). Experimental data are shown for $R=400 \mathrm{~nm}$ (circles; cf. Fig. 9), $R=655 \mathrm{~nm}$ (squares; cf. Fig. 10), and $R=1180 \mathrm{~nm}$ (triangles; cf. Fig. 11).

$\alpha$ show no agreement, as exemplarily plotted for $\alpha=0$ (solid circles).

In Fig. 12, the experimentally determined spurious force obtained by the difference between the forces derived with the

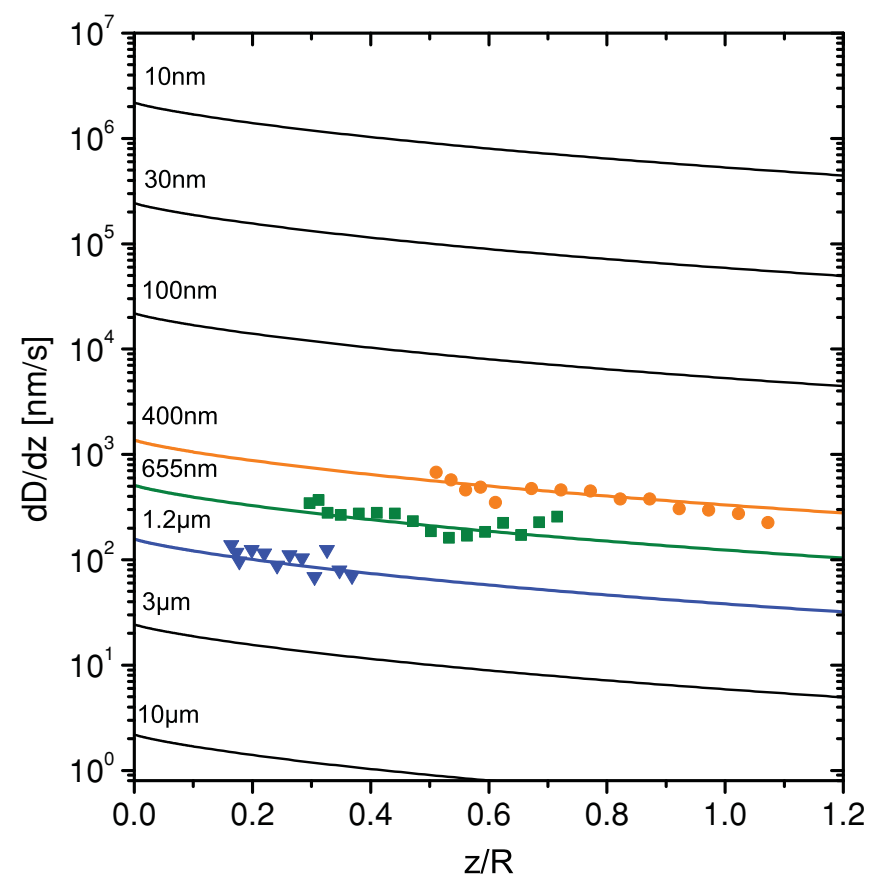

FIG. 13. (Color online) Distance dependence of the spurious drift $\frac{d}{d z} D$ for various particle radii $R$ (solid lines). Experimental data are shown for $R=400 \mathrm{~nm}$ (circles; cf. Fig. 9), $R=655 \mathrm{~nm}$ (squares; cf. Fig. 10), and $R=1180 \mathrm{~nm}$ (triangles; cf. Fig. 11). uncorrected drift method [Eq. (3) or (5) with $\alpha=0$ ] and the equilibrium potential method [Eq. (1)] is plotted for different particle sizes as a function of $z / R$. The data are in good agreement with the solid lines representing the theoretical predictions for the spurious force $\alpha \gamma \frac{d}{d z} D(z)$ with $\alpha=1$. The spurious force depends only on the particle radius, but is independent of $\rho_{s}$. It increases for particles closer to the wall and for smaller particles due to the larger $D$, reaching values on the order of several piconewtons for particles with $R=10 \mathrm{~nm}$, the size of a macromolecule.

By dividing the spurious forces (Fig. 12) by $\gamma$, it is possible to derive the spurious drift $\alpha \frac{d}{d z} D(z)$, which is plotted in Fig. 13 for $\alpha=1$. The lines corresponding to the theoretical predictions and the symbols representing the experimental data for the particles with $R=400 \mathrm{~nm}$ (solid circles), $R=655 \mathrm{~nm}$ (squares), and $R=1080 \mathrm{~nm}$ (triangles) show good agreement. Due to the increasing gradient of the diffusion coefficient when approaching a surface, the spurious drift increases for shorter particle-wall distances and for smaller particles. In contrast to the spurious force, which diverges for small $z$, the spurious drift reaches a maximum at $z=0$.

\section{CONCLUSIONS}

The experiments presented in this paper clearly demonstrate that the presence of noise-induced drift has to be considered in force measurements based on the drift; neglecting the drift can lead to artifacts, which may even suggest the wrong sign of the force. Such spurious forces become more significant for smaller systems and may reach the piconewton range for objects the size of a biomolecule, i.e., about $10 \mathrm{~nm}$. Furthermore, we stress that a constant diffusion coefficient can be assumed only for a particle far from any boundary. Such boundaries are naturally introduced by surfaces or by other particles in suspension, a situation that is typically met in experiments.

\section{ACKNOWLEDGMENT}

We would like to thank Johanna Widoniak for the preparation of the titanium oxide particles.

\section{APPENDIX: MATHEMATICAL DESCRIPTION OF THE SPURIOUS DRIFT: STOCHASTIC DIFFERENTIAL EQUATIONS AND THE FOKKER-PLANCK EQUATION}

The motion of a Brownian particle can be described in several ways. In this Appendix we clarify the relations between the various approaches, putting them in a historical context and demonstrating how the spurious drift emerges. We refer to Ref. [21] for further details.

The first diffusion theory was developed by Smoluchowski [28] and, independently, by Einstein [29] at the beginning of the 20th century. The fundamental object of this theory was the transition probability density $p_{s, t}\left(z_{\text {initial }}, z_{\text {final }}\right)$ from position $z_{\text {initial }}$ to position $z_{\text {final }}$ between times $s$ and $t>s$. The individual particle trajectories played no major role in this description of the diffusion process. In the 1930s Kolmogorov showed that defining a probability measure on the path space is 
equivalent to specifying all finite-dimensional distributions of a stochastic process, i.e., all joint distributions of the random variables $z\left(t_{1}\right), z\left(t_{2}\right), \ldots, z\left(t_{k}\right)$, where $t_{1}<t_{2}<\ldots<t_{k}$ are some time instants (Kolmogorov's extension theorem; for a thorough mathematical treatment see, e.g., Ref. [30]). For the Markov processes, such as processes describing the motion of Brownian particles, finite-dimensional distributions are determined in turn by the initial distribution and by the transition probability density mentioned above. This shows, at an abstract level, an equivalence of Kolmogorov's general approach and the Smoluchowski-Einstein transition probability description.

While the Kolmogorov extension theorem restores the concept of an individual path to its position of central importance in the description of a diffusion process, it still leaves out another fundamental physical ingredient: infinitesimal evolution law. This is furnished by the theory of stochastic differential equations (SDEs), which describe the time evolution of individual paths. This theory, developed in the 1940s by Itô [21] (and to some extent also by Gikhman and Stratonovitch), presents diffusion as a motion of a particle according to a random dynamic law. Solutions to SDEs are Markov processes and, at least in principle, transition probabilities are determined by the equation and, as we have seen, they determine the statistics of the trajectories.

The preceding remarks are purported to explain the status of the Fokker-Planck equation [21] and its place in diffusion theory. This partial differential equation (in mathematics called the Kolmogorov forward equation) has the transition density as its solution. It is thus an infinitesimal approach to diffusion at the level of the transition densities. As explained above, knowing the solution to the Fokker-Planck equation gives one full knowledge of the diffusion process. It is thus of the utmost importance for a successful implementation of the Fokker-Planck equation method, given a SDE, to be able to produce the correct form of the Fokker-Planck equation from its coefficients. Before we address this point, we have to discuss the so-called Itô-Stratonovitch dilemma, which is at the root of the existence of the spurious drift and deals with the interpretation of a SDE [31].

\section{Stochastic differential equation approach}

We consider the one-dimensional, time-independent SDE

$$
d z=\frac{F(z)}{\gamma(z)} d t+\sqrt{2 D(z)} d W(t),
$$

where $W(t)$ denotes a Wiener process, i.e., a stochastic process, whose increments are stationary, independent, and normally distributed with $W(t)-W(s)$ having mean zero and variance equal to $|t-s|$. Such an equation describes, for example, the behavior of a Brownian particle in the presence of a position-dependent diffusion coefficient $D(z)$. While only onedimensional SDEs are discussed here, these considerations can be straightforwardly generalized to the multidimensional case.

Equation (A2) should be interpreted as the integral equation [21],

$$
z(T)=\int_{0}^{T} \frac{F(z)}{\gamma(z)} d t+\int_{0}^{T} \sqrt{2 D(z)} d W(t) .
$$

However, due to the irregularity of the Wiener process, as well as the solution $z(t)$ of the SDE, the second integral on the right-hand side has several possible interpretations. More precisely, it is defined as a limit of integral sums, $\sum_{n=0}^{N-1} \sigma\left[z\left(t_{j}^{*}\right)\right]\left[W\left(t_{j+1}\right)-W\left(t_{j}\right)\right]$, where $t_{j}$ are points dividing the interval $[0, T]$ into $N$ equal subintervals and the intermediate points are defined by $t_{j}^{*}=(1-\alpha) t_{j}+\alpha t_{j+1}$. A crucial point is that the value of the limit, i.e., of the stochastic integral, depends on the choice of $\alpha$. Thus, in every problem, in addition to the SDE, we have to know the value of $\alpha$ for the mathematical model of the phenomenon under investigation to be well defined. Common choices are $\alpha=0$ (the Itô convention), $\alpha=0.5$ (the Stratonovitch convention), and $\alpha=$ 1 (the isothermal, anti Itô, or backward Itô convention).

The different choices of $\alpha$ are connected to each other by a precise mathematical relationship. Namely, the above SDE with a given choice of $\alpha$ is equivalent to the Itô equation $(\alpha=0)$

$$
d z=\frac{F(z)}{\gamma(z)} d t+\alpha \frac{d D(z)}{d z} d t+\left.\sqrt{2 D(z)} d W\right|_{\alpha=0},
$$

that is, an equation with any choice of $\alpha$ can be rewritten equivalently as an Itô equation, at the cost of adding an

TABLE II. Correct use of the SDE and the Fokker-Planck equation for systems coupled to a heat bath.

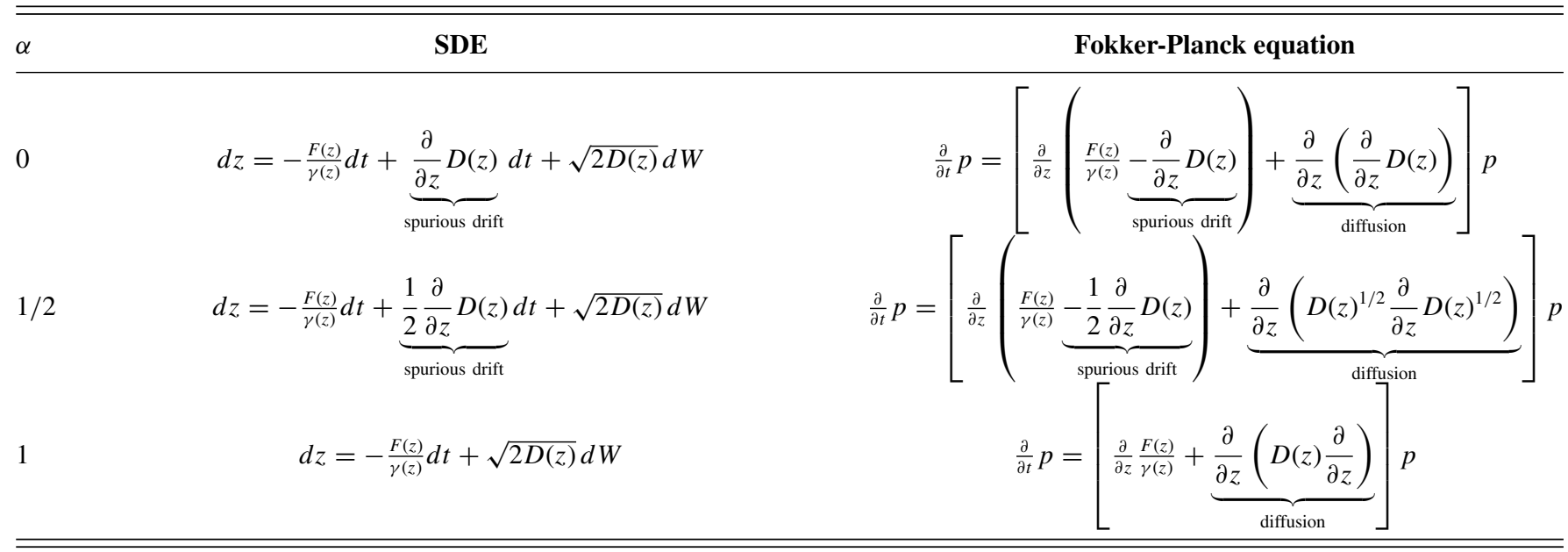


additional term $\alpha \frac{d D(z)}{d z} d t$. This term has been called "spurious drift" $-\mathrm{a}$ confusing name since it may suggest its nonphysical character, while, as we have seen, this additional drift term may be fully observable in a real physical situation [9].

Concisely, we see here the central problem addressed in this paper: The choice of $\alpha$ leads, in the Itô form of the equation, to an extra drift term, which vanishes only when the diffusion coefficient is constant. When $D$ is position dependent, at most one of these values of $\alpha$ can correctly describe the system; such a parameter may depend on the system under investigation. However, if the system is coupled to a heat bath, as is the case for a Brownian particle, the spurious drift and the associated spurious force are maximal, i.e., $\alpha=1$ in Eq. (A3). Of course, as shown in Table II, other conventions may also be used by adjusting the weight of the spurious drift term; remarkably, for the anti Itô or isothermal convention the correction term vanishes [22].

\section{Fokker-Planck approach}

Since the Fokker-Planck equation is deterministic, its solution involves no randomness and is thus uniquely determined. However, given a SDE, the prescription for writing the associated Fokker-Planck equation depends on the convention adopted, as shown in Table II. To guarantee that the dynamics reproduces equilibrium properties correctly when the noise term is interpreted with a given $\alpha$ (first column), the SDE (second column) must be corrected with an additional drift term. The corresponding Fokker-Planck equation (third column) obtained from the SDE contains a drift term, which also includes the corresponding spurious drift and a diffusion term, which is commonly written in various forms in the literature. We note that each form corresponds to a different choice of $\alpha$ for the interpretation of the noise in the underlying SDE, such that the Fokker-Planck equation is unique, i.e., the sum of the extra term due to the spurious drift and the diffusion term is constant.
[1] E. M. Purcell, Am. J. Phys. 45, 3 (1977).

[2] J. Happel and H. Brenner, Low Reynolds Number Hydrodynamics (Springer, New York, 1983).

[3] D. L. Ermak and J. A. McCammon, J. Chem. Phys. 69, 1352 (1978).

[4] D. Ryter, Z. Phys. B: Condens. Matter 41, 39 (1981).

[5] J. M. Sancho, M. San Miguel, and D. Duerr, J. Stat. Phys. 28, 291 (1982).

[6] A. T. Clark, M. Lal, and G. M. Watson, Faraday Discuss. Chem. Soc. 83, 179 (1987).

[7] P. Lançon, G. Batrouni, L. Lobry, and N. Ostrowsky, Europhys. Lett. 54, 28 (2001).

[8] S. H. Behrens, J. Plewa, and D. Grier, Eur. Phys. J. E 10, 115 (2003).

[9] G. Volpe, L. Helden, T. Brettschneider, J. Wehr, and C. Bechinger, Phys. Rev. Lett. 104, 170602 (2010).

[10] A. D. Mehta, M. Rief, J. A. Spudich, D. A. Smith, and R. M. Simmons, Science 283, 1689 (1999).

[11] C. Bustamante, Z. Bryant, and S. B. Smith, Nature (London) 421, 423 (2003).

[12] G. Binnig, C. F. Quate, and C. Gerber, Phys. Rev. Lett. 56, 930 (1986).

[13] L. P. Ghislain and W. W. Webb, Opt. Lett. 18, 1678 (1993).

[14] K. Berg-Sørensen and H. Flyvbjerg, Rev. Sci. Instrum. 75, 594 (2004).

[15] G. Volpe, G. Volpe, and D. Petrov, Phys. Rev. E 76, 061118 (2007).
[16] J. Walz, Curr. Opin. Colloid Interface Sci. 2, 600 (1997).

[17] D. C. Prieve, Adv. Colloid Interface Sci. 82, 93 (1999).

[18] G. Volpe, L. Helden, T. Brettschneider, and C. Bechinger, Opt. Express 17, 23975 (2009).

[19] V. Blickle, T. Speck, C. Lutz, U. Seifert, and C. Bechinger, Phys. Rev. Lett. 98, 210601 (2007).

[20] V. Blickle, T. Speck, U. Seifert, and C. Bechinger, Phys. Rev. E 75, 060101 (2007)

[21] B. Øksendal, Stochastic Differential Equations (Springer, Heidelberg, 2007).

[22] A. W. C. Lau and T. C. Lubensky, Phys. Rev. E 76, 011123 (2007).

[23] R. Kupferman, G. A. Pavliotis, and A. M. Stuart, Phys. Rev. E 70, 036120 (2004).

[24] S. Eiden Assmann, J. Widoniak, and G. Maret, Chem. Mater. 16, 6 (2004).

[25] M. A. Bevan and D. C. Prieve, Langmuir 15, 7925 (1999).

[26] H. H. von Grünberg, L. Helden, P. Leiderer, and C. Bechinger, J. Chem. Phys. 114, 10094 (2001).

[27] H. Brenner, Chem. Eng. Sci. 16, 242 (1961).

[28] M. Smoluchowski, Ann. Phys. (Leipzig) 326, 756 (1906).

[29] A. Einstein, Investigations on the Theory of Brownian Movement (Dover, New York, 1956).

[30] R. Durrett, Probability: Theory and Examples, 4th ed. (Cambridge University Press, Cambridge, 2010).

[31] N. van Kampen, Stochastic Processes in Physics and Chemistry, 3rd ed. (North-Holland, Amsterdam, 2007). 